\title{
Tinnitus and tinnitus disorder
}

Citation for published version (APA):

De Ridder, D., Schlee, W., Vanneste, S., Londero, A., Weisz, N., Kleinjung, T., Shekhawat, G. S., Elgoyhen, A. B., Song, J-J., Andersson, G., Adhia, D., de Azevedo, A. A., Baguley, D. M., Biesinger, E., Binetti, A. C., Del Bo, L., Cederroth, C. R., Cima, R., Eggermont, J. J., ... Langguth, B. (2021). Tinnitus and tinnitus disorder: Theoretical and operational definitions (an international multidisciplinary proposal). Progress in Brain Research, 260, 1-25. https://doi.org/10.1016/bs.pbr.2020.12.002

\section{Document status and date:}

Published: 01/01/2021

DOI:

10.1016/bs.pbr.2020.12.002

Document Version:

Publisher's PDF, also known as Version of record

\section{Document license:}

Taverne

\section{Please check the document version of this publication:}

- A submitted manuscript is the version of the article upon submission and before peer-review. There can be important differences between the submitted version and the official published version of record.

People interested in the research are advised to contact the author for the final version of the publication, or visit the DOI to the publisher's website.

- The final author version and the galley proof are versions of the publication after peer review.

- The final published version features the final layout of the paper including the volume, issue and page numbers.

Link to publication

\footnotetext{
General rights rights.

- You may freely distribute the URL identifying the publication in the public portal. please follow below link for the End User Agreement:

www.umlib.nl/taverne-license

Take down policy

If you believe that this document breaches copyright please contact us at:

repository@maastrichtuniversity.nl

providing details and we will investigate your claim.
}

Copyright and moral rights for the publications made accessible in the public portal are retained by the authors and/or other copyright owners and it is a condition of accessing publications that users recognise and abide by the legal requirements associated with these

- Users may download and print one copy of any publication from the public portal for the purpose of private study or research.

- You may not further distribute the material or use it for any profit-making activity or commercial gain

If the publication is distributed under the terms of Article $25 \mathrm{fa}$ of the Dutch Copyright Act, indicated by the "Taverne" license above, 


\section{Tinnitus and tinnitus} disorder: Theoretical and operational definitions

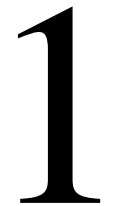
(an international multidisciplinary proposal)

Dirk De Ridder ${ }^{\mathrm{a}, *}$, Winfried Schlee ${ }^{\mathrm{b}}$, Sven Vanneste ${ }^{\mathrm{c}}$, Alain Londero ${ }^{\mathrm{d}}$, Nathan Weisz ${ }^{\mathrm{e}}$, Tobias Kleinjung ${ }^{\mathrm{f}}$, Giriraj Singh Shekhawat ${ }^{\mathrm{g}, \mathrm{h}, \mathrm{i}}{ }^{\mathrm{i}}$,

Ana Belén Elgoyhen ${ }^{\mathrm{j}}$, Jae-Jin Song ${ }^{\mathrm{k}}$, Gerhard Andersson ${ }^{1, \mathrm{~m}}$, Divya Adhia ${ }^{a}$, Andreia Aparecida de Azevedo ${ }^{\mathrm{n}}$, David M. Baguley ${ }^{\mathrm{o}, \mathrm{p}}$, Eberhard Biesinger ${ }^{\mathrm{q}}$, Ana Carolina Binetti ${ }^{r}$, Luca Del Bo ${ }^{\mathrm{s}}$, Christopher R. Cederroth ${ }^{\mathrm{p}, \mathrm{t}, \mathrm{u}}$, Rilana Cima ${ }^{\mathrm{v}, \mathrm{w}, \mathrm{x}}$, Jos J. Eggermont ${ }^{y}$, Ricardo Figueiredo ${ }^{z, a a}$, Thomas E. Fuller ${ }^{v, a b}$, Silvano Gallus ${ }^{\text {ac }}$, Annick Gilles $^{\text {ad,ae }}$, Deborah A. Hall ${ }^{\text {af,ag,ah,ai }}$ (1), Paul Van de Heyning ${ }^{\text {aj }}$ (D), Derek J. Hoare ${ }^{\text {ak }}$, Eman M. Khedral ${ }^{\text {a }}$, Dimitris Kikidis ${ }^{\text {am }}$, Maria Kleinstaeuber ${ }^{\text {an }}$, Peter M. Kreuzer ${ }^{\mathrm{b}}$, Jen-Tsung Lai ${ }^{\mathrm{a} o}$, Jose Miguel Lainez ${ }^{\mathrm{ap}}{ }$, Michael Landgrebe ${ }^{\mathrm{aq}}{ }^{\mathrm{\prime}}$, Lieber Po-Hung Li ${ }^{\text {ar }}$, Hubert H. Lim ${ }^{\text {as }}$, Tien-Chen Liu ${ }^{\text {at }}$, Jose Antonio Lopez-Escamez ${ }^{\mathrm{au}, \mathrm{av}, \mathrm{aw}}$, Birgit Mazurek ${ }^{\mathrm{ax}}$, Aage R. Molleray', Patrick Neff ${ }^{\text {az }}$, Christo Pantev ${ }^{\text {ba }}$, Shi Nae Park ${ }^{\text {bb }}$, Jay F. Piccirillo ${ }^{\text {bc }}$, Timm B. Poeppl ${ }^{\text {bd }}$, Josef P. Rauschecker ${ }^{\text {be,bf }}$, Richard Salvi ${ }^{\text {bg }}$, Tanit Ganz Sanchez ${ }^{\mathrm{bh}, \mathrm{bi}}$, Martin Schecklmann ${ }^{\mathrm{b}}$, Axel Schiller ${ }^{\mathrm{b}}$, Grant D. Searchfield ${ }^{\mathrm{bj}, \mathrm{bk}, \mathrm{bl}}$, Richard Tyler ${ }^{\mathrm{bm}}$, Veronika Vielsmeier ${ }^{\mathrm{bn}}$, Johan W.S. Vlaeyen ${ }^{\mathrm{w}, \mathrm{bo}}$, Jinsheng Zhang ${ }^{\mathrm{bp}}$, Yiwen Zheng ${ }^{\mathrm{bq}}$, Matteo de Nora ${ }^{\mathrm{i}}$, and Berthold Langguth

${ }^{a}$ Department of Surgical Sciences, Section of Neurosurgery, Dunedin School of Medicine, University of Otago, Dunedin, New Zealand

${ }^{\mathrm{b}}$ Department of Psychiatry and Psychotherapy, Bezirksklinikum, University of Regensburg, Regensburg, Germany ${ }^{\mathrm{c}}$ Lab for Clinical and Integrative Neuroscience, Global Brain Health Institute, Trinity College Institute of Neuroscience, Trinity College Dublin, Ireland

${ }^{\mathrm{d}}$ Service ORL CCF, Hôpital Européen Georges Pompidou, Paris, France ${ }^{\mathrm{e}}$ Centre for Cognitive Neuroscience, University of Salzburg, Salzburg, Austria ${ }^{\mathrm{f}}$ Department of Otorhinolaryngology—Head and Neck Surgery, University Hospital Zurich, University of Zurich, Zurich, Switzerland

${ }^{\mathrm{g}}$ College of Nursing and Health Sciences, Flinders University, Bedford Park, SA, Australia

${ }^{\mathrm{h}}$ Ear Institute, University College London, London, United Kingdom

${ }^{\mathrm{i}}$ Tinnitus Research Initiative, Regensburg, Germany 
${ }^{\mathrm{j}}$ Instituto de Investigaciones en Ingeniería Genética y Biología Molecular "Dr. Héctor N. Torres" (INGEBI), Buenos Aires, AR, Argentina

${ }^{\mathrm{k}}$ Department of Otorhinolaryngology, Head and Neck Surgery, Seoul National University College of Medicine, Seoul National University Bundang Hospital, Seongnam, Republic of Korea ${ }^{1}$ Department of Behavioural Sciences and Learning, Linköping University, Linköping, Sweden

${ }^{\mathrm{m}}$ Department of Biomedical and Clinical Sciences, Linköping University, Linköping, Sweden ${ }^{\mathrm{n}}$ OTOSUL, otorrinolaringologia Sul Fluminense, Volta Redonda, Brazil

${ }^{\circ}$ Otology and Hearing Group, Division of Clinical Neuroscience, School of Medicine, University of Nottingham, Nottingham, United Kingdom

${ }^{\mathrm{p}}$ National Institute for Health Research (NIHR) Nottingham Biomedical Research Centre, Nottingham University Hospitals NHS Trust, Nottingham, United Kingdom ${ }^{\mathrm{q}}$ Centre of Otorhinolaryngology, Private Practice, Traunstein, Germany ${ }^{\mathrm{r}}$ Buenos Aires British Hospital, Buenos Aires, Argentina

${ }^{\mathrm{s}}$ Del Bo Tecnologia per l'ascolto srl, Milan, Italy

${ }^{\mathrm{t}}$ Hearing Sciences, Division of Clinical Neuroscience, School of Medicine, University of Nottingham, Nottingham, United Kingdom

${ }^{\mathrm{u}}$ Laboratory of Experimental Audiology, Department of Physiology and Pharmacology, Karolinska Institutet, Stockholm, Sweden

${ }^{\mathrm{v}}$ Department of Clinical Psychological Science, Maastricht University, Maastricht, The Netherlands

${ }^{\mathrm{w}}$ Department of Health Psychology, Katholieke Universiteit Leuven, Leuven, Belgium

${ }^{\mathrm{x}}$ Adelante, Centre of Expertise in Rehabilitation and Audiology, Hoensbroek, The Netherlands ${ }^{\mathrm{y}}$ Department of Psychology, Department of Physiology and Pharmacology, University of Calgary, Calgary, $A B$, Canada

${ }^{\mathrm{z}}$ Centro Universitário de Valença, Faculdade de Medicina, Valença, Brazil

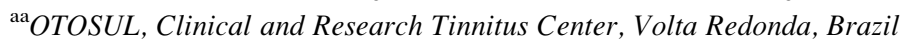
${ }^{\mathrm{ab}}$ Spine and Biologics, Medtronic, Maastricht, Netherlands

${ }^{\mathrm{ac}}$ Laboratory of Lifestyle Epidemiology, Department of Environmental Health Sciences, Istituto di Ricerche Farmacologiche Mario Negri IRCCS, Milan, Italy

${ }^{\mathrm{ad}}$ Department of otorhinolaryngology and Head \& Neck Surgery, Antwerp University Hospital, Edegem, Belgium

${ }^{\text {ae }}$ Faculty of Medicine, Antwerp University, Wilrijk, Belgium

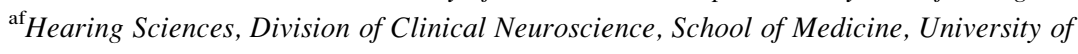
Nottingham, Nottingham, United Kingdom

${ }^{\mathrm{ag}}$ National Institute for Health Research (NIHR) Nottingham Biomedical Research Centre, University of Nottingham, Nottingham, United Kingdom ${ }^{\mathrm{ah}}$ Nottingham University Hospitals NHS Trust, Queens Medical Centre, Nottingham, United Kingdom

${ }^{\text {ai } U n i v e r s i t y ~ o f ~ N o t t i n g h a m ~ M a l a y s i a, ~ S e m e n y i h, ~ M a l a y s i a ~}$

${ }^{\mathrm{aj}}$ Univ Dept of Otorhinolaryngology and head and neck surgery, Antwerp University Hospital, Department of Translational Neurosciences, University of Antwerp. Antwerpen, Belgium ${ }^{\mathrm{ak}}$ NIHR Nottingham Biomedical Research Centre, Hearing Sciences, Division of Clinical Neuroscience, University of Nottingham, Nottingham, United Kingdom

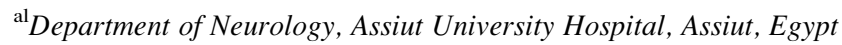
${ }^{\mathrm{am}} 1$ st Department of Otolaryngology-Head and Neck Surgery, National and Kapodistrian University of Athens, Athens, Greece 


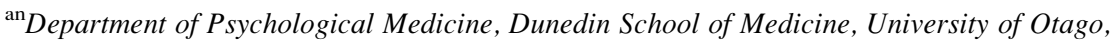
Dunedin, New Zealand ${ }^{\text {ao } D e p a r t m e n t ~ o f ~ O t o l a r y n g o l o g y, ~ K u a n g-T i e n ~ G e n e r a l ~ H o s p i t a l, ~ T a i c h u n g, ~ T a i w a n ~}$

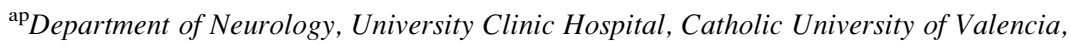
Valencia, Spain ${ }^{\mathrm{aq}}$ kbo-Lech-Mangfall-Kliniken Agatharied, Hausham, Germany

${ }^{\text {ar }}$ Department of Otolaryngology, Cheng Hsin General Hospital, Taipei, Taiwan

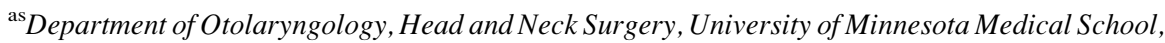
University of Minnesota, Minneapolis, MN, United States

${ }^{a t}$ Department of Otolaryngology, National Taiwan University, Taipei, Taiwan ${ }^{\text {au } O t o l o g y ~ \& ~ N e u r o t o l o g y ~ G r o u p ~ C T S ~ 495, ~ D e p a r t m e n t ~ o f ~ G e n o m i c ~ M e d i c i n e, ~ G E N Y O . ~ C e n t r e ~ f o r ~}$ Genomics and Oncological Research: Pfizer/University of Granada/Andalusian Regional Government, PTS Granada, Granada, ES, Spain ${ }^{a v}$ Department of Otolaryngology, Instituto de Investigación Biosanitaria Ibs. Granada, Hospital Universitario Virgen de las Nieves, Granada, ES, Spain ${ }^{\mathrm{aw}}$ Department of Surgery, Division of Otolaryngology, Universidad de Granada, Granada, ES, Spain ${ }^{\mathrm{ax}}$ Tinnitus Center, Charité-Universitätsmedizin Berlin, Berlin, Germany ${ }^{a y}$ School of Behavioral and Brain Sciences, The University of Texas at Dallas, Dallas, TX, United States

${ }^{\mathrm{az}}$ University Research Priority Program Dynamics of Healthy Aging, University of Zurich, Zurich, Switzerland

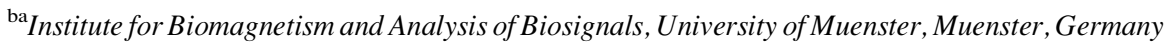

${ }^{\mathrm{bb}}$ Department of Otorhinolaryngology —Head and Neck Surgery, College of Medicine, The Catholic University of Korea, Seoul, South Korea ${ }^{\mathrm{bc}}$ Department of Otolaryngology—Head and Neck Surgery, Washington University School of Medicine, St. Louis, MO, United States ${ }^{\mathrm{bd}}$ Department of Psychiatry, Psychotherapy and Psychosomatics, Faculty of Medicine, RWTH Aachen University, Aachen, Germany

${ }^{b e}$ Department of Neuroscience, Georgetown University, Washington, DC, United States ${ }^{\mathrm{bf}}$ Hans Fischer Senior Fellow and TUM Ambassador, Institute for Advanced Study and Klinikum Rechts der Isar, TUM, Munich, Germany ${ }^{\text {bg }}$ SUNY Distinguished Professor, Center for Hearing and Deafness, 137 Cary Hall, University at Buffalo, Buffalo, NY, United States

${ }^{\mathrm{bh}}$ Department of Otolaryngology, University of São Paulo School of Medicine, São Paulo, Brazil

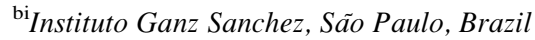

${ }^{\mathrm{bj}}$ Section of Audiology, The University of Auckland, Auckland, New Zealand

${ }^{\mathrm{bk}}$ Eisdell Moore Centre, The University of Auckland, Auckland, New Zealand ${ }^{\mathrm{bl}}$ Centre for Brain Research, The University of Auckland, Auckland, New Zealand ${ }^{\mathrm{bm}}$ Department of Communication Sciences and Disorders, Department of Otolaryngology-Head and Neck Surgery, Iowa City, IA, USA



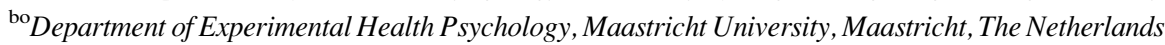
${ }^{\mathrm{bp}}$ Department of Communication Sciences \& Disorders, Department of Otolaryngology, Wayne State University, Detroit, MI, United States ${ }^{\mathrm{bq}}$ Department of Pharmacology and Toxicology, University of Otago, Dunedin, New Zealand *Corresponding author:Tel.: +64275601144,e-mail address:dirk.deridder@otago.ac.nz 


\section{Abstract}

As for hypertension, chronic pain, epilepsy and other disorders with particular symptoms, a commonly accepted and unambiguous definition provides a common ground for researchers and clinicians to study and treat the problem. The WHO's ICD11 definition only mentions tinnitus as a nonspecific symptom of a hearing disorder, but not as a clinical entity in its own right, and the American Psychiatric Association's DSM-V doesn't mention tinnitus at all.

Here we propose that the tinnitus without and with associated suffering should be differentiated by distinct terms: "Tinnitus" for the former and "Tinnitus Disorder" for the latter. The proposed definition then becomes "Tinnitus is the conscious awareness of a tonal or composite noise for which there is no identifiable corresponding external acoustic source, which becomes Tinnitus Disorder "when associated with emotional distress, cognitive dysfunction, and/or autonomic arousal, leading to behavioural changes and functional disability.". In other words "Tinnitus" describes the auditory or sensory component, whereas "Tinnitus Disorder" reflects the auditory component and the associated suffering. Whereas acute tinnitus may be a symptom secondary to a trauma or disease, chronic tinnitus may be considered a primary disorder in its own right.

If adopted, this will advance the recognition of tinnitus disorder as a primary health condition in its own right. The capacity to measure the incidence, prevalence, and impact will help in identification of human, financial, and educational needs required to address acute tinnitus as a symptom but chronic tinnitus as a disorder.

\section{Keywords}

Tinnitus, Phantom, Sound, Definition, Affective, Pain, Operational, Theoretical

\section{Introduction}

Tinnitus is a clinical symptom associated with changes in auditory and other brain systems with a similar prevalence in different geographical regions: estimates for the general adult population indicate that prevalence of any current tinnitus ranges from $11.9 \%$ to $30.3 \%$ (McCormack et al., 2016). Other data provide an estimate about the prevalence of stressful tinnitus (Davis and El Rafaie, 2000). In that study the prevalence of tinnitus was $10.1 \%$, of which $0.5 \%$ were very severely distressed, $1 \%$ severely distressed and 5\% experienced at least "moderate" distress (Davis and El Rafaie, 2000).

An indispensable precondition for both clinical and scientific research is a clear and commonly accepted definition of a clinical term. Moreover, such a definition is essential for correct placement of a disorder within the existing classification systems such as the International Classification of Disorders (ICD) or the Diagnostic and Statistical Manual of Mental Disorders (DSM). A commonly accepted definition is a requirement for epidemiological research, for estimation of the global health and economic cost of tinnitus, as well as for health insurances and health care regulators. The recent shift toward web-based research, accelerated by the COVID19 pandemic, also sets a wider context for considering the development of a standardized definition. 
The current lack of a commonly accepted definition of tinnitus is problematic for the purposes of population and web-based surveys (e.g., Biswas et al., 2019; Simoes et al., 2019), and the lack of commonly accepted clinical criteria is problematic for the purpose of identifying patient groups.

Moreover, existing definitions of tinnitus do not differentiate between people who experience tinnitus and those who experience tinnitus together with tinnitus-associated emotional distress, cognitive dysfunction, autonomic arousal and functional disability.

For the purpose of clinical research, the degree of suffering is most relevant, but there are no commonly accepted clinical diagnostic criteria for quantifying tinnitusrelated suffering and the functional disability associated with that suffering. In chronic pain, which is commonly considered the somatosensory analogue of chronic tinnitus (De Ridder et al., 2007, 2011a; De Ridder and Moller, 2011; De Ridder and Van de Heyning, 2007; Llinas et al., 1999; Moller, 1997, 2000, 2007a; Rauschecker et al., 2015; Tonndorf, 1987; Vanneste et al., 2018a, 2019) it has been shown that the perceived disability of the pain is only weakly correlated with pain intensity (Doualla et al., 2019; Garbi Mde et al., 2014), but strongly with catastrophic misinterpretations of pain (Kovacs et al., 2011; Severeijns et al., 2001), which determine the painrelated suffering (Wade et al., 2011). In the tinnitus field, it has also been shown that the quality of life is predicted by tinnitus catastrophizing and tinnitus-related fear, rather than the psychophysical measures of tinnitus (Cima et al., 2011).

Several efforts have been made over the last decades to quantify the severity of tinnitus by assessing the tinnitus-related emotional distress and handicap level, either by self-report questionnaires or by structured interviews (Andersson et al., 1999; Douek, 1981; McCombe et al., 2001). In spite of these efforts, a commonly accepted definition for tinnitus and its varying degree of emotional distress, cognitive dysfunction, autonomic arousal and functional disability is still lacking, and no diagnostic criteria have been established for the different forms of tinnitus.

Therefore, the Tinnitus Research Initiative (TRI), a non-profit organization supporting scientific and clinical research with a special focus on a collaborative multidisciplinary and international approach (TRI, 2020) has taken the lead in initiating a continuing process of defining tinnitus and its associated clinical signs and symptoms. The start of this ongoing endeavor involves a proposal for tinnitus, endorsed by an international multidisciplinary group of tinnitus experts.

\section{Current situation: Commonly used definitions, characteristics and diagnostic classification}

Multiple definitions of tinnitus have been published, from "ringing or buzzing in the ears" (Oxford Dictionary), to "the conscious experience of a sound that originates in the head of its owner" (McFadden, 1982) or "the conscious perception of an auditory sensation in the absence of a corresponding external stimulus" (Baguley et al., 2013; Elgoyhen et al., 2015; Langguth et al., 2013; Moller, 2007b). These are combined in the ICD11 definition. In ICD 11, under MC41 tinnitus is described as "A nonspecific 
symptom of hearing disorder characterized by the sensation of buzzing, ringing, clicking, pulsations, and other noises in the ear in the absence of appropriate corresponding external stimuli and in the absence of what the examiner can hear with a stethoscope" (ICD11, 2019). None of these definitions is more commonly used than another. "Ringing in the ears" is clearly too simplistic - and frequently people perceive the origin of the phantom sound not in the ears, but in the head or even outside the head. Furthermore, tinnitus is not always described as "ringing" but also as a hissing sound, or cricket-like sound and many more descriptions. Some definitions are more comprehensive descriptors of tinnitus, but are unhelpful because they include musical hallucinosis (Vanneste et al., 2013) or auditory hallucinations, (e.g., the perception of voices in schizophrenia) and do not differentiate between etiologically different types of tinnitus, namely subjective and objective tinnitus. The term "objective tinnitus" (or its synonym "somatosound") is used to describe sounds that are generated by a sound source in the body, for example, by muscle contractions or blood flow.

Similarly, the existing definitions do not distinguish between people who are particularly sensitive to sound, e.g., people who perceive low-frequency noise which is present (in the environment) but not perceived by others (Frosch, 2016), and those who perceive a low-frequency phantom sound, i.e., a sound which is not present in the environment.

As with pain, tinnitus consists not only of a sensory component, often expressed as tinnitus loudness and frequency or pitch, but also an affective component, reflecting its unpleasantness (De Ridder et al., 2011a) and its related distress (De Ridder et al., 2011b). Tinnitus associated distress involves an autonomic component (van der Loo et al., 2011), and should be differentiated from mood changes, with different neural correlates (Joos et al., 2012). Similarly to pain, suffering is the result from a dysfunctional cognitive, emotional and autonomic response (Craig, 2003; Wade and Hart, 2002; Wade et al., 2011). For example, this could include depression, and anxiety, as well as sleep disturbances, concentration, attention, and memory problems (Baguley et al., 2013; Langguth, 2011). These complaints can lead to behavioral changes and functional disability, even though not everybody with tinnitus suffers from it. In order to quantify the impact of tinnitus on quality of life, several selfreport questionnaires have been developed and validated. These are used to stratify tinnitus patients according to the severity of their perceived tinnitus and also as outcome measurement for clinical trials (Langguth et al., 2007).

Tinnitus is not only heterogeneous with respect to its clinical comorbidities. The pathophysiology of tinnitus can be heterogeneous as well, as tinnitus can be a secondary symptom of specific pathologies, such as Meniere's disease, otosclerosis or vestibular schwannoma. The majority of patients presenting with tinnitus have hearing loss measurable with conventional pure tone audiometry (Langguth et al., 2017; Moller, 2007b; Norena et al., 2002). And even in tinnitus patients with normal standard pure tone audiometry there may be some form of hearing loss (Barnea et al., 1990; Frosch, 2016; Stouffer and Tyler, 1990; Vanneste et al., 2013; Weisz et al., 2006). 
With more sophisticated investigations cochlear dysfunction can be detected even in the presence of a normal audiogram. These include high frequency audiometry (Barnea et al., 1990; Hallmo et al., 1995; Vielsmeier et al., 2015), distortion product otoacoustic emissions (DPOAE) (Dadoo et al., 2019), threshold equalizing in noise (TEN) testing (Weisz et al., 2006) or auditory evoked potentials (Schaette and McAlpine, 2011). Recently the term "hidden hearing loss" has been introduced to describe auditory symptoms such as tinnitus or poor speech discrimination in combination with a normal pure tone audiometry, a pattern that could be related to cochlear synaptopathy, as shown in animal studies (Barbee et al., 2018). If no specific etiology is identified, the tinnitus is sometimes also called tinnitus "e causa ignota" or "idiopathic" tinnitus.

Finally, tinnitus is not very well represented in current classification systems. The abovementioned ICD11 definition focuses exclusively on the auditory component of tinnitus. Also the ICD 11 criterion "....in the absence of what the examiner can hear with a stethoscope" is problematic as not all cases of pulsatile tinnitus, tensor tympani and stapedius myoclonus can be detected by a stethoscope. In DSM versions IV and $\mathrm{V}$, tinnitus is not yet represented at all.

\subsection{Why is there no consensus definition?}

To date, there is neither a standardized theoretical nor operational definition of tinnitus. Theoretical definitions define a term in a scientific discipline, functioning as a proposal to see a phenomenon in a certain way (Wikipedia, 2020a). A theoretical definition is derived from a theory that includes causal predictions, and that can change, based on new evidence (Wikipedia, 2020a). An operational definition on the other hand describes in a pragmatic way what is meant by a certain term, such that the term is concrete, unambiguous, and measurable during clinical diagnosis and treatment. Operational definitions can also change with new insights.

Different reasons may account for the absence of a theoretical definition for tinnitus: first, there is no generally accepted pathophysiological model of tinnitus; second, there are different forms of tinnitus, and third, tinnitus is studied by many different scientific disciplines, each with their own theoretical framework. As is the case in pain science, the science of tinnitus is an extremely multidisciplinary field, with researchers and clinicians from various specializations, including audiologists, psychologists, ENT specialists, neurologists, psychiatrists, psychosomatic specialists, neurosurgeons, pharmacologists, neuroradiologists, basic and computational neuroscientists and many more. Although multidisciplinarity is an obvious strength, it also makes the process of reaching consensus of what tinnitus is and how it should be defined challenging.

The current paper can best be considered a starting point of an ongoing reflections and exchange of ideas, which ultimately could lead to a generally accepted definition of tinnitus. In this context, we accept that not all authors agree with every aspect of the proposed definitions as presented in Boxes 1 and 2. 


\section{BOX 1 Proposal for the definition of tinnitus and tinnitus disorder}

Tinnitus is the conscious awareness of a tonal or composite noise for which there is no identifiable corresponding external sound source.

Tinnitus Disorder is the conscious awareness of a tonal or composite noise for which there is no identifiable corresponding external sound source, associated with emotional and/or cognitive dysfunction, and/or autonomic arousal, leading to behavioral changes and functional disability. As a proposal for ICD coding this can be phrased in one sentence:

Tinnitus is the conscious awareness of a tonal or composite noise for which there is no identifiable corresponding external acoustic source, which becomes Tinnitus Disorder when associated with emotional distress, cognitive dysfunction, and/or autonomic arousal, leading to behavioral changes and functional disability.

Minimum time criterion: The tinnitus lasts for minimum $5 \mathrm{~min}$ per day and occurs on the majority of days.

Acute tinnitus: tinnitus with a duration of less than 3 months.

Chronic tinnitus: tinnitus with a duration of at least 3 months.

\section{BOX 2 Hypothetical pragmatic definition aligning with DSM diagnostic criteria for pain disorder}

According to the criteria for somatic symptom disorder specified as "with predominant tinnitus"

A. Tinnitus that is distressing or results in significant disruption of daily life.

B. Excessive thoughts, feelings, or behaviors related to tinnitus or associated health concerns as manifested by at least one of the following:

a. disproportionate and persistent thoughts about the seriousness of one's tinnitus,

b. persistently high level of anxiety about tinnitus.

c. excessive time and energy devoted to tinnitus.

C. Although the tinnitus sound may not be continuously present, the state of being symptomatic is persistent (typically more than 3 months).

Persistent: A persistent course is characterized by severe symptoms, marked impairment, and long duration (more than 3 months).

Mild: Only one of the symptoms specified in Criterion B is fulfilled.

Moderate: Two symptoms specified in Criterion B are fulfilled.

Severe: Two or more of the symptoms specified in Criterion B are fulfilled, plus there are multiple somatic complaints (or one very severe somatic symptom).

\subsection{The sensory and affective dimensions of tinnitus}

The proposed approach to differentiate between the sensory component and the associated symptoms is not new. Several decades ago, influential researchers already proposed a differentiation between tinnitus and the psychological reactions to the tinnitus (Jastreboff, 1990; Tyler et al., 1992). Neuroimaging research in the last decades enabled to differentiate between the neuronal signatures of the tinnitus percept and the associated affective aspects. The latter could be assigned to the activity or 
connectivity between brain regions often subsumed as the "limbic system," consisting of medial prefrontal and anterior cingulate cortex, and subcortical regions such as the ventral striatum, amygdala and hippocampus (Elgoyhen et al., 2015). Whether activity in limbic brain regions is a consequence of ("reaction to") the tinnitus sound or is one of the causes of tinnitus disorder, especially of its emotional sequelae/distress (similar to depression), or both, is still a matter of debate (Rauschecker et al., 2010). Even though there may be disagreement about some of the details of suggested models, such as the frontostriatal gating model, there is consensus about the fact that tinnitus disorders involve brain regions outside the auditory system. Reaching agreement about the participation of these structures and the nature of their interactions with the auditory system should be a major goal of future research on tinnitus.

A standardized set of questions for population based research on tinnitus has only very recently been developed (Biswas et al., 2019). For grading of tinnitus severity a large variety of different questionnaires were developed and are in use (Hall et al., 2016). Even if there is a relative high cross-correlation between the scores of these questionnaires, there is no commonly accepted definition for a diagnostic classification of tinnitus severity. The same holds true for outcome measures for the assessment of the efficacy of specific therapeutic interventions, even though some steps have been taken including a Delphi consensus method in order to come to a consensus among key stakeholders (Hall et al., 2018, 2019; Langguth et al., 2007; Michiels et al., 2018).

Perceptual aspects of tinnitus are either assessed by asking the patient or by matching tinnitus loudness and tinnitus pitch to external tones. Currently there exists no established objective behavioral and/or neural correlate. Such a marker would be highly desirable for defining or diagnosing the presence of tinnitus or for validating clinical measures. Currently there are some preliminary studies that provide an orientation, which clinical assessment instruments might be successful candidates. As an example, there are no neural correlates of the tinnitus loudness match, but there do exist neural correlates of subjectively perceived loudness as expressed on a visual analogue or numeric rating scale (De Ridder et al., 2015a). This fits with the finding that loudness measurement with visual analogue or numeric rating scales have much better test-retest reliability and construct validity than loudness match data (Hall et al., 2017).

\section{Requirements for a definition of tinnitus}

Ideally, a theoretical definition matches with the current knowledge of pathophysiology, and considers that tinnitus is a heterogeneous condition with respect to its perceptual characteristics, its etiological factors, and its comorbidities. Specific auditory phantom perceptions such as verbal hallucinations or musical hallucinosis should be differentiated from tinnitus, as well as the various forms of objective tinnitus and specific tinnitus subtypes with specific etiologic factors. 
Moreover, the definition also considers the time course of tinnitus and tinnitus disorder. First, there should be a minimal duration and a minimal frequency of occurrence, and exclude phenomena such as spontaneous brief tinnitus, which is a short-lasting phantom perception that occurs from time to time, is unilateral in most cases and resolves spontaneously (Oron et al., 2011). In some epidemiological studies a minimum duration of $5 \mathrm{~min}$ has been used for defining tinnitus (Davis, 1989; McCormack et al., 2016), and it should be present most days. This is reminiscent of the recent definitions of epilepsy and hypertension. Epilepsy is defined and thus diagnosed by more than 2 unprovoked seizures occurring more than $24 \mathrm{~h}$ apart (Fisher et al., 2014) and hypertension is defined as repeated measurements of high blood pressure (>140/90 mmHg) (Bergler-Klein, 2019).

Second, even if the mechanisms involved in the transition from acute to chronic tinnitus are still incompletely understood, there are good reasons to differentiate between acute and chronic tinnitus. Although there is also no clinical criterion that would determine that an individual's tinnitus has become chronic, there is some evidence that the mechanisms responsible for the generation of tinnitus and those involved in the maintenance of tinnitus, are not identical. One would also expect that there are therapeutic interventions, which are only effective in the acute stage and not in the chronic state, or vice versa. In the pain literature, 3 months duration separate between acute and chronic pain, as defined by the International Association for the Study of Pain (IASP) and the ICD11 (Treede et al., 2015). It may be reasonable to adopt 3 months as the criterion for chronic tinnitus.

Third, the experience of tinnitus sound is not the same as the experience of tinnitus sound with associated suffering. Many people experience tinnitus, but about $80 \%$ of them are not suffering from it; others are severely impaired by their tinnitus (Axelsson and Ringdahl, 1989). Neuroimaging has revealed that these two groups differ in their brain activity and brain connectivity pattern (Hullfish et al., 2018; Maudoux et al., 2012; Mohan et al., 2018a,b; Schecklmann et al., 2013; Ueyama et al., 2013; Vanneste and De Ridder, 2015; Vanneste et al., 2018b). Thus it seems not appropriate to subsume these two groups in the same diagnostic category.

Fourth, a definition that holds practical applications, allows with a reasonable effort to determine who has tinnitus and tinnitus disorder respectively and who does not. If this were possible by self-report, it would facilitate population based investigations and online surveys.

Fifth, the definition should be accepted and supported by different specialists from diverse fields such as basic neuroscience, audiology, otology, neuro-otology, epidemiology, neurology, neurosurgery, neurophysiology, psychiatry and psychology, who all approach tinnitus from a slightly different angle.

Sixth, a definition should use terminology that is understood by the general population. This is necessary for the communication with patients, relatives, all the different health care providers, policy makers, communities and international organizations and stakeholders.

Finally, a theoretical definition is not absolute and may be modified or revised in the future, once the symptom is better understood, and should thus not be a constraint for future research and treatment developments. 


\subsection{Tinnitus: Symptom or disorder}

A stimulus produces an effect on the different sensory receptors, inducing sensation (De Ridder et al., 2011a). Perception in the setting of tinnitus has been defined as the act of interpreting and organizing a sensory stimulus to produce a meaningful experience of the world and of oneself (De Ridder et al., 2011a). Furthermore, sensation and perception can be conscious or unconscious (Dehaene et al., 1998). Unconscious perception means that one responds to a stimulus even though one is not consciously aware of the stimulus, for example, in priming (Dehaene et al., 1998). Consciousness is a multifaceted concept that has two major components: awareness of environment and of self (i.e., the content of consciousness) and wakefulness (i.e., the level of consciousness) (Laureys, 2005; Norrsell, 1986). For tinnitus to be heard by a person, both wakefulness and awareness are required.

A first step in defining tinnitus relates to the acknowledgement that all individuals with tinnitus are consciously aware of a sound that (even if transient) has no acoustic source, i.e., for which there is no identifiable corresponding sound source externally or from inside one's body. The sound sensation of tinnitus can be tonal or noise-like, it can have a pattern, e.g., resemble the sounds of crickets or cicadas, but it has no specific meaning cognitively. This is in contrast to other auditory phantom percepts such as musical hallucinosis (Vanneste et al., 2013) and verbal hallucinations. These should best be excluded from the definition of tinnitus, even if transitions between tinnitus, musical and verbal hallucinations are described in the literature (Marneros et al., 1997).

Whereas musical hallucinosis is usually encountered in people with severe hearing loss, verbal hallucinations are typical for psychosis, as encountered in schizophrenia (Griffiths, 2000; Vanneste et al., 2013). Thus, a preliminary definition could be that "tinnitus is the conscious awareness of a constant or intermittent sound that has no intrinsic meaning and for which there is no identifiable corresponding external sound source." As such, tinnitus is a sign of dysfunctional auditory sensation and perception. Yet, analogous to chronic pain, tinnitus may have a suffering component, and then it can be defined as a disorder.

A disorder is a functional abnormality or disturbance (Wikipedia, 2020b). The term disorder is often considered more value-neutral and less stigmatizing than the terms disease or illness (Wikipedia, 2020b). In mental health, the term mental disorder is used as a way of acknowledging the complex interaction of biological, social, and psychological factors in psychiatric conditions (Wikipedia, 2020b). In other words, tinnitus disorder is the auditory component of tinnitus in combination with its associated affective component. Affect has been defined as the manner in which emotional events influence behavior and subjective feelings, often operationalized in terms of valence and arousal (Kragel and LaBar, 2016). Thus, affect incorporates emotion, mood, arousal or distress associated with the phantom sound. All of the above can either lead to pleasure, i.e., positive affect, or suffering, i.e., negative affect. Suffering can be defined as an unpleasant experience associated with negative cognitive, emotional and autonomic impact. Suffering is not an all or nothing problem, it is better to see this a gradient or a spectrum. If severe and associated with a 
lack of coping it can lead to psychiatric pathology but not necessarily so. The negative affective component is especially relevant when the sensation is annoying, unpredictable and uncontrollable. In chronic disabling tinnitus the negative affect predominates and thus tinnitus associated suffering. This leads to a definition of tinnitus disorder "tinnitus disorder is the conscious awareness of a tonal or composite noise for which there is no identifiable corresponding external acoustic source, with associated suffering."

Suffering is a broad term, which has been well studied in the field of somatic symptom disorder (SSD), and may thus be extrapolated to the tinnitus domain. The DSM-5 criteria of SSD (Katz et al., 2015) refer to three main components of psychological distress associated with the somatic symptom: (1) disproportionate thoughts, (2) high levels of anxiety or other negative emotions, (3) excessive time and energy devoted to the symptom including dysfunctional symptom-related behaviors (Katz et al., 2015). These reflect cognitive (thoughts), affective or emotional and autonomic reactions (energy) to the somatic symptom, whether pain, tinnitus or other somatic symptoms, analogous to the above definition of suffering. In a recently published high-quality systematic review of psychosocial correlates of tinnitus distress all three domains of SSD mentioned above were covered (Trevis et al., 2018). According to the DSM-5 the severity of the SSD is graded according to the number of the mentioned symptoms (Mild: Only one of the symptoms specified in Criterion B is fulfilled; Moderate: Two symptoms specified in Criterion B are fulfilled; Severe: Two or more of the symptoms specified in Criterion B are fulfilled, plus there are multiple somatic complaints (or one very severe somatic symptom)). As this procedure seems to be rather arbitrary, it is recommended to complement it by using validated self-report instruments for assessing the individual tinnitus severity. Also a grading with a simple screening question "How much of a problem is your tinnitus on a scale between 1 and 5" have been proven valid and useful (Zeman et al., 2014).

\subsection{Relevant aspects for the definition and diagnostic classification of tinnitus}

\subsubsection{Objective and subjective tinnitus}

Even though objective and subjective tinnitus may be unfortunate terms, and since long abandoned in physical medicine and pain research (Rothstein, 1996), the definitions should be inclusive of both objective and subjective tinnitus.

Both definitions, Tinnitus and Tinnitus Disorder can indeed be applied to objective and subjective tinnitus. It suffices that "external" in the definition actually means "external to the body," and as such "tinnitus is the conscious awareness of a constant or intermittent sound that has no intrinsic meaning and for which there is no identifiable corresponding external (to the body) sound source." Objective tinnitus is then described as tinnitus that can be attributed to an internal sound source, whereas subjective tinnitus is tinnitus with no reference to an internal sound source. As somatosounds, which lead to objective tinnitus, are generated within the body by muscular contraction or vascular bruits, this entity is included by the definition. In the same 
way, pulsatile tinnitus is captured by this definition. Yet, it should be evident that mechanistically, somatosounds are not phantom-sound-like percepts: somatosounds are sounds mechanically produced by the body, in contrast to subjective tinnitus, where there is neither an external nor an internal sound source. The pathophysiology is entirely different. In subjective tinnitus there is no corresponding sound source, whereas in somatosounds there is. From a pathophysiological point of view somatosounds and most forms of pulsatile (excluding somatosensory pulsatile tinnitus (Levine et al., 2008)) might not fall under the term tinnitus, but from a phenomenological point of view for the patient and a clinical point of view for the health care provider they may, as patients seek help for both problems from the same clinicians. Moreover, in many cases the perceptual characteristics suggest an internal sound source even if the exact source cannot be identified. An example is pulsesynchronous tinnitus in which a vascular origin (hypertension, dehiscence, fistula, tumor, aneurysm, stenosis, etc.) is expected, but is not identifiable by the respective diagnostic tests. Also in tinnitus with typical characteristics of muscular origin it is sometimes difficult to differentiate the exact etiology (middle ear muscles palatal muscles, etc.)

Typically these form of tinnitus are described by additional adjectives, such as objective, pulsatile or other.

There is some criticism about the terms "subjective tinnitus" and "objective tinnitus." The terms are somewhat misleading, as objectively measurable neuronal correlates of subjective tinnitus are being developed (Liu et al., 2019; Vanneste et al., 2018a). Moreover the term "subjective tinnitus" might suggest, that the tinnitus does not really exist, but is imagined by the patient. Alternative terms for "subjective tinnitus" and "objective tinnitus" could be "primary tinnitus" and "secondary tinnitus." Naming and defining a coherent set of terms for subjective/primary and objective/secondary tinnitus, and incorporating somatosounds, arterial and venous pulsatile tinnitus may benefit from another group effort, convened by an interdisciplinary task force of tinnitus specialists, analogous to the IASP task force.

Similarly to the ICD11 coding of different subtypes of pain, tinnitus subtypes may be defined. The proposed ICD11 classification for chronic pain differentiates neuropathic pain of peripheral and central origin. It comprises nine common conditions associated with chronic pain (Scholz et al., 2019; Treede et al., 2019). A similar approach should be taken for tinnitus.

\subsection{Acute and chronic tinnitus}

For the differentiation between acute and chronic tinnitus typically a duration of 3-6 months is chosen. However, there is no empirical evidence either for these time points. Also sometimes tinnitus of recent onset (Vanneste et al., 2011; Weise et al., 2013) and persistent tinnitus (Langguth et al., 2010) is used. Acute tinnitus could be considered a secondary symptom of an underlying problem, such as traumatic or non-traumatic hearing loss, metabolic, nutritional, infectious, genetic, autoimmune, and/or vascular processes. If it becomes chronic, tinnitus may become a primary 
disorder in its own right, analogous to the concept in pain (Treede et al., 2019). Chronic pain was defined previously as pain that persists past normal healing time, and hence lacks the acute warning function of physiological nociception (Treede et al., 2019), but since its release by the WHO in 2018 of ICD11, is now simplified based on a temporal criterion of 3 months (Scholz et al., 2019; Treede et al., 2015, 2019). It is generally accepted that the mechanisms involved in the development of tinnitus differ from the mechanisms involved in the maintenance of tinnitus, but the dynamics of this transition are still largely unclear. Moreover, neuroimaging research suggests that changes occur in brain activity and connectivity gradually with increasing tinnitus duration (Schecklmann et al., 2013; Schlee et al., 2009; Vanneste et al., 2011).

In this context also the terms "peripheral tinnitus" and "central tinnitus" are sometimes used (Eggermont, 2003; Gerken, 1996; Sahley and Nodar, 2001; Risey et al., 1995). Peripheral tinnitus refers to the auditory perception that results from aberrant neural activity at the cochlear level and is transmitted through the auditory pathways (Guitton et al., 2003; Haider et al., 2018; Jastreboff, 1990; Puel and Guitton, 2007). Central tinnitus refers to the auditory perception that is generated in auditory brain centers by the aberrant neural activity and is sustained by that aberrant neural activity (Eggermont, 2005, 2007; Haider et al., 2018; Kaltenbach, 2006, 2007; Norena, 2011). Despite this distinction, peripheral tinnitus and central tinnitus are not completely independent: the transition from acute to chronic tinnitus is described as the "centralisation" of an initially peripheral tinnitus (Eggermont, 2003). The same terminology is used in pain, and incorporated in the IASP and ICD11 definitions for pain, in which peripheral and central pain are differentiated (Scholz et al., 2019; Treede et al., 2019). The question is whether this is relevant as tinnitus likely can only become consciously perceived by the brain, i.e., by central involvement (De Ridder et al., 2011a, 2015b; Ortmann et al., 2011; van der Loo et al., 2009). On the other hand it may determine whether a peripheral or central treatment approach is preferred, as shown in pain. In phantom pain, in about half of the patients a plexus block can transiently abolish pain, whether in the other half it doesn't result in any benefit. This has been described as centralization of pain (Birbaumer et al., 1997).

In addition, the temporal aspects of when the auditory phantom sensation turns into a psychological/psychiatric problem, i.e., a disorder, are currently still incompletely understood. It differs among patients, whether the suffering starts simultaneously with the auditory sensation of tinnitus or whether the suffering develops gradually and stabilizes after a certain moment in time, and whether once stable it could be considered chronic or persistent and turn into a disorder. In other words, whereas tinnitus at onset may be a symptom that is associated with, e.g., fear, when it becomes associated with pathological anxiety or depression, it becomes a disorder.

From a clinical perspective, and analogous to former definitions of chronicity in pain, a differentiation between acute and chronic tinnitus could be guided by the chance of spontaneous recovery or by the efficacy of specific interventions for acute tinnitus, but also here there exist no convincing data. Two small studies have found 
that of those patients who present within 4 weeks less than $20 \%$ become tinnitus free, suggesting that chronification already sets in earlier (Wallhausser-Franke et al., 2017; Vielsmeier et al., 2020). In those patients with sudden sensorineural hearing loss associated with tinnitus most patients with moderate hearing loss become tinnitus-free by day 7 confirming previous data, yet in more severe hearing loss improvement is more gradual starting after 1 month. About $2 / 3(65.2 \%)$ of mild to moderate hearing loss become tinnitus free within 3 months (in contrast to $22.7 \%$ of severe hearing loss), suggesting that acute tinnitus can resolve spontaneously within the acute time frame (Muhlmeier et al., 2016).

This study is in agreement with a 3 month cut-off as defined in chronic pain and thus 3 months could be proposed as a demarcation, based on a simple temporal criterion, between acute and chronic tinnitus, albeit without hard scientific proof, but more on the pathophysiological, clinical and treatment analogies between tinnitus and pain (De Ridder and Van de Heyning, 2007; De Ridder et al., 2011a; Llinas et al., 1999; Moller, 1997, 2000, 2007a; Rauschecker et al., 2015; Tonndorf, 1987; Vanneste et al., 2018a, 2019).

\section{Measurement of tinnitus}

Tinnitus is most often a lived experience, currently without a quantifiable measure, independent of the subjective expression of the tinnitus patient. This has to be considered in the development of an operational definition of tinnitus. From an audiological perspective it has been shown that weak or no correlation exists between changes in psychoacoustic measurements (matched tinnitus loudness or pitch) and changes in self-reported tinnitus associated suffering, and importantly, the changes in selfreported suffering, but not the change in psychoacoustic measurements, correlate with global subjective improvement (Hall et al., 2017) or the effect size of a treatment (Rabau et al., 2015).

Currently no objective biomarker has been established, either for the existence of tinnitus, or for the degree of suffering. Artificial intelligence using support vector machine learning on structural MRI brain scans (Liu et al., 2019) as well as electroencephalographic measurements (Vanneste et al., 2018a) have shown some promise by discriminating people with tinnitus from those without tinnitus with a specificity and sensitivity of about $80 \%$. However, validation of these data, and a higher accuracy is required before either fMRI or EEG can be used as a reliable diagnostic test.

Yet, the accuracy of identifying suffering from electroencephalographic data is a lot more difficult. Depression, as one of the types of suffering, can only be detected with $75 \%$ accuracy, using the same machine learning approach (Vanneste et al., 2018a). To complicate matters, the brain areas that determine or exclude the presence of tinnitus represent areas involved both in the sensory and affective components of tinnitus such as the auditory cortex and rostral to dorsal anterior cingulate cortex, as well as the parahippocampal area (Vanneste et al., 2018a). Indeed, tinnitus has been described as a unifying percept of multiple dynamically interactive overlapping 
networks (De Ridder et al., 2014). Thus currently, reliable biomarkers for the sensory and the affective components of tinnitus are not yet available.

In the absence of an ability to have a quantifiable measure the sensory and affective components independent of the subjectively expressed tinnitus percept, an operational definition based on biomarkers is not possible. However, this does not mean that an operational definition of tinnitus cannot be put forward. For many disorders that are primarily characterized by their subjective experience, there exist established operational definitions in DSM or ICD.

\section{Proposal for diagnostic criteria of tinnitus and tinnitus disorder}

"Tinnitus" can be defined as "the conscious awareness of a tonal or composite noise for which there is no identifiable corresponding external acoustic source."

By contrast "tinnitus disorder" can best be defined as "tinnitus plus tinnitusassociated emotional and/or cognitive dysfunction, and/or autonomic arousal leading to behavioural changes and functional disability."

Combining the two definitions in one sentence it becomes: "Tinnitus is the conscious awareness of a tonal or composite noise for which there is no identifiable corresponding external acoustic source, which becomes Tinnitus Disorder when associated with emotional distress, cognitive dysfunction, and/or autonomic arousal, leading to behavioural changes and functional disability"

Tinnitus definitions include objective and subjective, pulsatile and non-pulsatile, constant and intermittent, acute and chronic, but exclude more complex auditory phantom percepts such as musical hallucinosis and auditory or verbal hallucinations.

\section{Conclusion}

In sum, a theoretical definition can be proposed that differentiates tinnitus without and with suffering. Whereas the first can be defined as "tinnitus is the conscious awareness of a tonal or composite noise for which there is no identifiable corresponding external sound source," the latter is defined as "tinnitus disorder is tinnitus with associated suffering." These definitions include objective and pulsatile tinnitus, but exclude more complex auditory phantom percepts such as musical hallucinosis and verbal hallucinations.

\section{Outlook and future perspectives}

The Tinnitus Research Initiative (TRI) is a multidisciplinary, international research initiative exclusively addressing tinnitus and associated auditory dysfunctions, such hyperacusis, misophonia, phonophobia and others. Based on the well-known analogy 
between chronic tinnitus and chronic pain, the TRI would be well placed to create a taskforce to further develop the definitions for all these terms, and to evaluate the usefulness, benefits and disadvantages of aligning the definitions with existing classification and coding systems, such as the WHO's ICD and the American Psychiatric Association's DSM. The major advantage would be that tinnitus could become recognized as an entity and/or disorder in its own right.

As an example, the current description of tinnitus in the ICD may benefit from some adjustments. The WHO, via the ICD11 defines tinnitus as "A nonspecific symptom of hearing disorder characterized by the sensation of buzzing, ringing, clicking, pulsations, and other noises in the ear in the absence of appropriate corresponding external stimuli and in the absence of what the examiner can hear with a stethoscope."

This definition is suboptimal for many reasons. First of all, chronic tinnitus is not only a symptom, but possibly a disorder in its own right, as discussed above. Secondly, tinnitus can occur in the absence of a clinically detectable hearing disorder. Thirdly, there are many other kinds of sounds that people with tinnitus hear, such as sea or electricity-like noise, crickets etc. And fourth, this definition excludes what is now still called objective tinnitus, pulsatile tinnitus and somatosounds. It can be argued that this should indeed not be incorporated under the term tinnitus, but for the reasons mentioned earlier we do suggest that for now included in the definition.

In order to make the ICD11 definition align more with the current definitions proposed in this manuscript it could be rephrased as follows: Tinnitus is the conscious awareness of a tonal or composite noise for which there is no identifiable corresponding external acoustic source, which when associated with emotional distress, cognitive dysfunction, and/or autonomic arousal, leading to behavioral changes and functional disability becomes a disorder.

Whereas most tinnitus specialists would agree on aligning with the WHO, not everybody would agree to align a definition with the DSM. The task force could investigate the advantages and disadvantages of collaborating with the American Psychiatry Association to evaluate the usefulness of incorporating a definition of tinnitus in the DSM. A pragmatic approach could for example be to align the diagnostic criteria for tinnitus disorder with those developed for pain disorder, a part of Somatic Symptom Disorder in DSM-5.

The diagnostic criteria for Somatic Symptom Disorder noted in DSM 5 are:

A. One or more somatic symptoms that are distressing or result in significant disruption of daily life.

B. Excessive thoughts, feelings, or behaviors related to the somatic symptoms or associated health concerns as manifested by at least one of the following:

- disproportionate and persistent thoughts about the seriousness of one's symptoms,

- persistently high level of anxiety about health or symptoms,

- excessive time and energy devoted to these symptoms or health concerns.

C. Although any one somatic symptom may not be continuously present, the state of being symptomatic is persistent (typically more than 6 months). 
The somatic symptom disorder can be specified as "with predominant pain" (previously pain disorder) for individuals whose somatic symptoms predominantly involve pain.

Based on the pathophysiological, clinical and treatment analogies between chronic pain and chronic tinnitus it is proposed that tinnitus disorder falls under the somatic symptom disorder specified as "with predominant tinnitus". The other specifications could also be maintained:

Persistent: A persistent course is characterized by severe symptoms, marked impairment, and long duration (more than 6 months; Criterion C) $>$ it would make sense to change this into 3 months (see above).

Mild: Only one of the symptoms specified in Criterion B is fulfilled.

Moderate: Two or more of the symptoms specified in Criterion B are fulfilled.

Severe: Two or more of the symptoms specified in Criterion B are fulfilled, plus there are multiple somatic complaints (or one very severe somatic symptom).

Aligning a DSM classification with pain and other somatic symptom disorders makes sense for multiple reasons: (1) The pathophysiology, clinical picture, co-morbidities and some forms of treatments of tinnitus and pain show similarities (De Ridder and Van de Heyning, 2007; De Ridder et al., 2011a; Llinas et al., 1999; Moller, 1997, 2000, 2007a; Rauschecker et al., 2015; Tonndorf, 1987; Vanneste et al., 2018a, 2019). (2) It makes it easier to extend tinnitus research to other disciplines and thereby grow interest in tinnitus research. (3) It permits a simple adaptation of the DSM to recognize and integrate tinnitus as a clinical entity.

It could be questioned whether the grading in tinnitus in mild, moderate and severe according to the presence of 1,2 or 3 or the symptoms in criterion B is valuable, and that tinnitus specific questionnaires should be used. Yet, the simplicity of this approach has its merits. The first symptom under criterion $\mathrm{B}$, a disproportionate and persistent thoughts about the seriousness of one's tinnitus, reflects a dysfunctional cognitive response to the phantom sound, whereas the second symptom, namely a persistently high level of anxiety about health or symptoms reflects more an emotional response, and the third symptom, excessive (time and) energy devoted to the tinnitus more an autonomic and behavioral response, it does reflect a layered response and thus some grading to the severity. Of course many patients with excessive thoughts will also become anxious or vice versa, and inherently spent much time paying attention to their tinnitus, but a pragmatic approach suggests that the benefits of proposing this definition may outweigh the negatives.

A major criticism on the proposal to align the tinnitus disorder definition with the DSM is that it seems to imply that tinnitus disorder would become a psychiatric diagnosis and that psychiatrists would become the main health care providers for tinnitus disorder. Furthermore, the grading is deemed insufficient. While these are understandable objections, the example of chronic pain may somewhat moderate these remarks. Chronic pain is also coded within the DSM, and most patients are treated by pain physicians, not psychiatrists. Eventually the classification of chronic pain in the DSM may have contributed to advances in pain management in the sense, that patients are treated by multidisciplinary teams, which involve also psychologists 
and psychiatrists. Such an approach might be desirable for tinnitus as well. Furthermore, most pain patients would not consider themselves psychiatric and, analogous to tinnitus, there exist questionnaires to grade pain associated suffering.

A consensus on definitions for tinnitus may facilitate acceptance of tinnitus as a disorder in its own right within the DSM classification. Yet it is up to a task force and a wider consensus within the tinnitus research community to decide on whether ICD and DSM definitions would be beneficial or not. In addition, the capacity to measure the incidence, prevalence, and impact will help in identification of human, financial, and educational needs required to address acute tinnitus as a symptom and chronic tinnitus as a disorder. This outweighs the imperfections of the grading within the current classification, which is being revised every couple of years anyway, depending on developing and novel insights.

An operational definition of "tinnitus" and some of its subforms are presented in Box 1. A hypothetical pragmatic definition of "tinnitus disorder" is proposed as an example of how this definition could be based on the current DSM 5 "somatic symptom disorder," analogous to pain, but then specified as tinnitus (see Box 2).

The subdivision between tinnitus as a sound percept without a corresponding external sound source and tinnitus disorder, which is tinnitus with its associated suffering, will be beneficial both for clinicians and researchers.

\section{Acknowledgments}

We would like to thankfully acknowledge the very valuable contributions to the manuscript by the following colleagues, who opted not to be listed as co-authors as they do not agree with all statements put forward in this manuscript:

Arnaud Norena, Pim van Dijk, Lucy Handscomb, James Henry, Fatima T. Husain, Margaret Jastreboff, Pawel J. Jastreboff, Elisabeth Marks, Laurence McKenna, Eui Cheol Nam, and William Sedley.

We are looking forward to further fruitful discussions on a comprehensive definition of tinnitus in the future.

\section{References}

Andersson, G., Lyttkens, L., Larsen, H.C., 1999. Distinguishing levels of tinnitus distress. Clin. Otolaryngol. Allied Sci. 24, 404-410.

Axelsson, A., Ringdahl, A., 1989. Tinnitus—a study of its prevalence and characteristics. Br. J. Audiol. 23, 53-62.

Baguley, D., McFerran, D., Hall, D., 2013. Tinnitus. Lancet 382, 1600-1607.

Barbee, C.M., James, J.A., Park, J.H., Smith, E.M., Johnson, C.E., Clifton, S., et al., 2018. Effectiveness of auditory measures for detecting hidden hearing loss and/or cochlear synaptopathy: a systematic review. Semin. Hear. 39, 172-209.

Barnea, G., Attias, J., Gold, S., Shahar, A., 1990. Tinnitus with normal hearing sensitivity: extended high-frequency audiometry and auditory-nerve brain-stem-evoked responses. Audiology 29, 36-45. 
Bergler-Klein, J., 2019. What's new in the ESC 2018 guidelines for arterial hypertension: the ten most important messages. Wien. Klin. Wochenschr. 131, 180-185.

Birbaumer, N., Lutzenberger, W., Montoya, P., Larbig, W., Unertl, K., Topfner, S., et al., 1997. Effects of regional anesthesia on phantom limb pain are mirrored in changes in cortical reorganization. J. Neurosci. 17, 5503-5508.

Biswas, R., Lugo, A., Gallus, S., Akeroyd, M.A., Hall, D.A., 2019. Standardized questions in English for estimating tinnitus prevalence and severity, hearing difficulty and usage of healthcare resources, and their translation into 11 European languages. Hear. Res. 377, 330-338.

Cima, R.F., Crombez, G., Vlaeyen, J.W., 2011. Catastrophizing and fear of tinnitus predict quality of life in patients with chronic tinnitus. Ear Hear. 32, 634-641.

Craig, A.D., 2003. A new view of pain as a homeostatic emotion. Trends Neurosci. 26, 303-307.

Dadoo, S., Sharma, R., Sharma, V., 2019. Oto-acoustic emissions and brainstem evoked response audiometry in patients of tinnitus with normal hearing. Int. Tinnitus J. 23, 17-25.

Davis, A.C., 1989. The prevalence of hearing impairment and reported hearing disability among adults in Great Britain. Int. J. Epidemiol. 18, 911-917.

Davis, A., El Rafaie, A., 2000. Epidemiology of tinnitus. In: Tyler, R. (Ed.), Tinnitus Handbook. Thomson Learning, San Diego, USA, pp. 1-24.

De Ridder, D., Moller, A., 2011. Similarities between treatments of tinnitus and central pain. In: Moller, A., Langguth, B., De Ridder, D., Kleinjung, T. (Eds.), Textbook of Tinnitus. Springer, New York, pp. 753-762.

De Ridder, D., Van de Heyning, P., 2007. The Darwinian plasticity hypothesis for tinnitus and pain. Prog. Brain Res. 166, 55-60.

De Ridder, D., De Mulder, G., Menovsky, T., Sunaert, S., Kovacs, S., 2007. Electrical stimulation of auditory and somatosensory cortices for treatment of tinnitus and pain. Prog. Brain Res. 166, 377-388.

De Ridder, D., Elgoyhen, A.B., Romo, R., Langguth, B., 2011a. Phantom percepts: tinnitus and pain as persisting aversive memory networks. Proc. Natl. Acad. Sci. U. S. A. 108, 8075-8080.

De Ridder, D., Vanneste, S., Congedo, M., 2011b. The distressed brain: a group blind source separation analysis on tinnitus. PLoS One 6, e24273.

De Ridder, D., Vanneste, S., Weisz, N., Londero, A., Schlee, W., Elgoyhen, A.B., et al., 2014. An integrative model of auditory phantom perception: tinnitus as a unified percept of interacting separable subnetworks. Neurosci. Biobehav. Rev. 44, 16-32.

De Ridder, D., Congedo, M., Vanneste, S., 2015a. The neural correlates of subjectively perceived and passively matched loudness perception in auditory phantom perception. Brain Behav., e00331.

De Ridder, D., Vanneste, S., Langguth, B., Llinas, R., 2015b. Thalamocortical dysrhythmia: a theoretical update in tinnitus. Front. Neurol. 6, 124.

Dehaene, S., Naccache, L., Le Clec, H.G., Koechlin, E., Mueller, M., Dehaene-Lambertz, G., et al., 1998. Imaging unconscious semantic priming. Nature 395, 597-600.

Doualla, M., Aminde, J., Aminde, L.N., Lekpa, F.K., Kwedi, F.M., Yenshu, E.V., et al., 2019. Factors influencing disability in patients with chronic low back pain attending a tertiary hospital in sub-Saharan Africa. BMC Musculoskelet. Disord. 20, 25.

Douek, E., 1981. Classification of tinnitus. Ciba Found. Symp. 85, 4-15.

Eggermont, J.J., 2003. Central tinnitus. Auris Nasus Larynx 30 (Suppl), S7-12. 
Eggermont, J.J., 2005. Tinnitus: neurobiological substrates. Drug Discov. Today 10, $1283-1290$.

Eggermont, J.J., 2007. Pathophysiology of tinnitus. Prog. Brain Res. 166, 19-35.

Elgoyhen, A.B., Langguth, B., De Ridder, D., Vanneste, S., 2015. Tinnitus: perspectives from human neuroimaging. Nat. Rev. Neurosci. 16, 632-642.

Fisher, R.S., Acevedo, C., Arzimanoglou, A., Bogacz, A., Cross, J.H., Elger, C.E., et al., 2014. ILAE official report: a practical clinical definition of epilepsy. Epilepsia 55, 475-482.

Frosch, F.G., 2016. Manifestations of a low-frequency sound of unknown origin perceived worldwide, also known as "the hum" or the "Taos hum". Int. Tinnitus J. 20, 59-63.

Garbi Mde, O., Hortense, P., Gomez, R.R., da Silva Tde, C., Castanho, A.C., Sousa, F.A., 2014. Pain intensity, disability and depression in individuals with chronic back pain. Rev. Lat. Am. Enfermagem 22, 569-575.

Gerken, G.M., 1996. Central tinnitus and lateral inhibition: an auditory brainstem model. Hear. Res. 97, 75-83.

Griffiths, T.D., 2000. Musical hallucinosis in acquired deafness. Phenomenology and brain substrate. Brain 123 (Pt. 10), 2065-2076.

Guitton, M.J., Caston, J., Ruel, J., Johnson, R.M., Pujol, R., Puel, J.L., 2003. Salicylate induces tinnitus through activation of cochlear NMDA receptors. J. Neurosci. 23, 3944-3952.

Haider, H.F., Bojic, T., Ribeiro, S.F., Paco, J., Hall, D.A., Szczepek, A.J., 2018. Pathophysiology of subjective tinnitus: triggers and maintenance. Front. Neurosci. 12, 866.

Hall, D.A., Haider, H., Szczepek, A.J., Lau, P., Rabau, S., Jones-Diette, J., et al., 2016. Systematic review of outcome domains and instruments used in clinical trials of tinnitus treatments in adults. Trials 17, 270.

Hall, D.A., Mehta, R.L., Fackrell, K., 2017. How to choose between measures of tinnitus loudness for clinical research? A report on the reliability and validity of an investigatoradministered test and a patient-reported measure using baseline data collected in a phase IIa drug trial. Am. J. Audiol. 26, 338-346.

Hall, D.A., Smith, H., Hibbert, A., Colley, V., Haider, H.F., Horobin, A., et al., 2018. The COMiT'ID study: developing core outcome domains sets for clinical trials of sound-, psychology-, and pharmacology-based interventions for chronic subjective tinnitus in adults. Trends Hear. 22, 2331216518814384.

Hall, D.A., Hibbert, A., Smith, H., Haider, H.F., Londero, A., Mazurek, B., et al., 2019. One size does not fit all: developing common standards for outcomes in early-phase clinical trials of sound-, psychology-, and pharmacology-based interventions for chronic subjective tinnitus in adults. Trends Hear. 23, 2331216518824827.

Hallmo, P., Borchgrevink, H.M., Mair, I.W., 1995. Extended high-frequency thresholds in noise-induced hearing loss. Scand. Audiol. 24, 47-52.

Hullfish, J., Abenes, I., Kovacs, S., Sunaert, S., De Ridder, D., Vanneste, S., 2018. Functional connectivity analysis of fMRI data collected from human subjects with chronic tinnitus and varying levels of tinnitus-related distress. Data Brief 21, 779-789.

ICD11, 2019. Tinnitus. https://icd.who.int/ct11/icd11_mms/en/release.

Jastreboff, P.J., 1990. Phantom auditory perception (tinnitus): mechanisms of generation and perception. Neurosci. Res. 8, 221-254.

Joos, K., Vanneste, S., De Ridder, D., 2012. Disentangling depression and distress networks in the tinnitus brain. PLoS One 7, e40544.

Kaltenbach, J.A., 2006. Summary of evidence pointing to a role of the dorsal cochlear nucleus in the etiology of tinnitus. Acta Otolaryngol. Suppl., 20-26. 
Kaltenbach, J.A., 2007. The dorsal cochlear nucleus as a contributor to tinnitus: mechanisms underlying the induction of hyperactivity. Prog. Brain Res. 166, 89-106.

Katz, J., Rosenbloom, B.N., Fashler, S., 2015. Chronic pain, psychopathology, and DSM-5 somatic symptom disorder. Can. J. Psychiatry 60, 160-167.

Kovacs, F.M., Seco, J., Royuela, A., Pena, A., Muriel, A., Spanish Back Pain Research Network, 2011. The correlation between pain, catastrophizing, and disability in subacute and chronic low back pain: a study in the routine clinical practice of the Spanish National Health Service. Spine 36, 339-345.

Kragel, P.A., LaBar, K.S., 2016. Decoding the nature of emotion in the brain. Trends Cogn. Sci. 20, 444-455.

Langguth, B., 2011. A review of tinnitus symptoms beyond 'ringing in the ears': a call to action. Curr. Med. Res. Opin. 27, 1635-1643.

Langguth, B., Goodey, R., Azevedo, A., Bjorne, A., Cacace, A., Crocetti, A., et al., 2007. Consensus for tinnitus patient assessment and treatment outcome measurement: tinnitus research initiative meeting, Regensburg, July 2006. Prog. Brain Res. 166, 525-536.

Langguth, B., Landgrebe, M., Wittmann, M., Kleinjung, T., Hajak, G., 2010. Persistent tinnitus induced by tricyclic antidepressants. J. Psychopharmacol. 24, 1273-1275.

Langguth, B., Kreuzer, P.M., Kleinjung, T., De Ridder, D., 2013. Tinnitus: causes and clinical management. Lancet Neurol. 12, 920-930.

Langguth, B., Landgrebe, M., Schlee, W., Schecklmann, M., Vielsmeier, V., Steffens, T., et al., 2017. Different patterns of hearing loss among tinnitus patients: a latent class analysis of a large sample. Front. Neurol. 8, 46.

Laureys, S., 2005. The neural correlate of (un)awareness: lessons from the vegetative state. Trends Cogn. Sci. 9, 556-559.

Levine, R.A., Nam, E.C., Melcher, J., 2008. Somatosensory pulsatile tinnitus syndrome: somatic testing identifies a pulsatile tinnitus subtype that implicates the somatosensory system. Trends Amplif. 12, 242-253.

Liu, Y., Niu, H., Zhu, J., Zhao, P., Yin, H., Ding, H., et al., 2019. Morphological neuroimaging biomarkers for tinnitus: evidence obtained by applying machine learning. Neural Plast. $2019,1712342$.

Llinas, R.R., Ribary, U., Jeanmonod, D., Kronberg, E., Mitra, P.P., 1999. Thalamocortical dysrhythmia: a neurological and neuropsychiatric syndrome characterized by magnetoencephalography. Proc. Natl. Acad. Sci. U. S. A. 96, 15222-15227.

Marneros, A., Beyenburg, S., Berghaus, A., 1997. Unilateral hallucinations and other psychotic symptoms due to otosclerosis. Psychopathology 30, 89-92.

Maudoux, A., Lefebvre, P., Cabay, J.E., Demertzi, A., Vanhaudenhuyse, A., Laureys, S., et al., 2012. Connectivity graph analysis of the auditory resting state network in tinnitus. Brain Res.

McCombe, A., Baguley, D., Coles, R., McKenna, L., McKinney, C., Windle-Taylor, P., et al., 2001. Guidelines for the grading of tinnitus severity: the results of a working group commissioned by the British Association of Otolaryngologists, head and neck surgeons, 1999. Clin. Otolaryngol. Allied Sci. 26, 388-393.

McCormack, A., Edmondson-Jones, M., Somerset, S., Hall, D., 2016. A systematic review of the reporting of tinnitus prevalence and severity. Hear. Res. 337, 70-79.

McFadden, D., 1982. Tinnitus: Facts, Theories, and Treatments. National Academy of Sciences Press, Washington DC.

Michiels, S., Ganz Sanchez, T., Oron, Y., Gilles, A., Haider, H.F., Erlandsson, S., et al., 2018. Diagnostic criteria for somatosensory tinnitus: a Delphi process and face-to-face meeting to establish consensus. Trends Hear. 22, 2331216518796403. 
Mohan, A., Alexandra, S.J., Johnson, C.V., De Ridder, D., Vanneste, S., 2018a. Effect of distress on transient network dynamics and topological equilibrium in phantom sound perception. Prog. Neuropsychopharmacol. Biol. Psychiatry 84, 79-92.

Mohan, A., De Ridder, D., Idiculla, R., C, D.S., Vanneste, S., 2018b. Distress-dependent temporal variability of regions encoding domain-specific and domain-general behavioral manifestations of phantom percepts. Eur. J. Neurosci. 48, 1743-1764.

Moller, A.R., 1997. Similarities between chronic pain and tinnitus. Am. J. Otol. 18, 577-585.

Moller, A.R., 2000. Similarities between severe tinnitus and chronic pain. J. Am. Acad. Audiol. 11, 115-124.

Moller, A.R., 2007a. Tinnitus and pain. Prog. Brain Res. 166, 47-53.

Moller, A.R., 2007b. Tinnitus: presence and future. Prog. Brain Res. 166, 3-16.

Muhlmeier, G., Baguley, D., Cox, T., Suckfull, M., Meyer, T., 2016. Characteristics and spontaneous recovery of tinnitus related to idiopathic sudden sensorineural hearing loss. Otol. Neurotol. 37, 634-641.

Norena, A.J., 2011. An integrative model of tinnitus based on a central gain controlling neural sensitivity. Neurosci. Biobehav. Rev. 35, 1089-1109.

Norena, A., Micheyl, C., Chery-Croze, S., Collet, L., 2002. Psychoacoustic characterization of the tinnitus spectrum: implications for the underlying mechanisms of tinnitus. Audiol. Neurootol. 7, 358-369.

Norrsell, U., 1986. Awareness, wakefulness and arousal. Acta Neurochir. Suppl. (Wien) 36, 86-88.

Oron, Y., Roth, Y., Levine, R.A., 2011. Sudden brief unilateral tapering tinnitus: prevalence and properties. Otol. Neurotol. 32, 1409-1414.

Ortmann, M., Muller, N., Schlee, W., Weisz, N., 2011. Rapid increases of gamma power in the auditory cortex following noise trauma in humans. Eur. J. Neurosci. 33, 568-575.

Puel, J.L., Guitton, M.J., 2007. Salicylate-induced tinnitus: molecular mechanisms and modulation by anxiety. Prog. Brain Res. 166, 141-146.

Rabau, S., Cox, T., Punte, A.K., Waelkens, B., Gilles, A., Wouters, K., et al., 2015. Changes over time of psychoacoustic outcome measurements are not a substitute for subjective outcome measurements in acute tinnitus. Eur. Arch. Otorhinolaryngol. 272, 573-581.

Rauschecker, J.P., leaver, A.M., Muhlau, M., 2010. Tuning out the noise: limbic-auditory interactions in tinnitus. Neuron 66, 819-826.

Rauschecker, J.P., May, E.S., Maudoux, A., Ploner, M., 2015. Frontostriatal gating of tinnitus and chronic pain. Trends Cogn. Sci. 19, 567-578.

Risey, J.A., Guth, P.S., Amedee, R.G., 1995. Furosemide distinguishes central and peripheral tinnitus. Int. Tinnitus J. 1, 99-103.

Rothstein, J.M., 1996. Objective versus subjective: kudzu terminology. Phys. Ther. $76,814-816$.

Sahley, T.L., Nodar, R.H., 2001. A biochemical model of peripheral tinnitus. Hear. Res. $152,43-54$.

Schaette, R., McAlpine, D., 2011. Tinnitus with a normal audiogram: physiological evidence for hidden hearing loss and computational model. J. Neurosci. 31, 13452-13457.

Schecklmann, M., Landgrebe, M., Poeppl, T.B., Kreuzer, P., Manner, P., Marienhagen, J., et al., 2013. Neural correlates of tinnitus duration and distress: a positron emission tomography study. Hum. Brain Mapp. 34, 233-240.

Schlee, W., Hartmann, T., Langguth, B., Weisz, N., 2009. Abnormal resting-state cortical coupling in chronic tinnitus. BMC Neurosci. 10, 11. 
Scholz, J., Finnerup, N.B., Attal, N., Aziz, Q., Baron, R., Bennett, M.I., et al., 2019. The IASP classification of chronic pain for ICD-11: chronic neuropathic pain. Pain 160, 53-59.

Severeijns, R., Vlaeyen, J.W., van den Hout, M.A., Weber, W.E., 2001. Pain catastrophizing predicts pain intensity, disability, and psychological distress independent of the level of physical impairment. Clin. J. Pain 17, 165-172.

Simoes, J., Neff, P., Schoisswohl, S., Bulla, J., Schecklmann, M., Harrison, S., et al., 2019. Toward personalized tinnitus treatment: an exploratory study based on internet crowd sensing. Front. Public Health 7, 157.

Stouffer, J.L., Tyler, R.S., 1990. Characterization of tinnitus by tinnitus patients. J. Speech Hear. Disord. 55, 439-453.

Tonndorf, J., 1987. The analogy between tinnitus and pain: a suggestion for a physiological basis of chronic tinnitus. Hear. Res. 28, 271-275.

Treede, R.D., Rief, W., Barke, A., Aziz, Q., Bennett, M.I., Benoliel, R., et al., 2015. A classification of chronic pain for ICD-11. Pain 156, 1003-1007.

Treede, R.D., Rief, W., Barke, A., Aziz, Q., Bennett, M.I., Benoliel, R., et al., 2019. Chronic pain as a symptom or a disease: the IASP classification of chronic pain for the international classification of diseases (ICD-11). Pain 160, 19-27.

Trevis, K.J., McLachlan, N.M., Wilson, S.J., 2018. A systematic review and meta-analysis of psychological functioning in chronic tinnitus. Clin. Psychol. Rev. 60, 62-86.

TRI, 2020. Tinnitus Research Initiative. https://www.tinnitusresearch.net/index.php.

Tyler, R.S., Aran, J.M., Dauman, R., 1992. Recent advances in tinnitus. Am. J. Audiol. $1,36-44$.

Ueyama, T., Donishi, T., Ukai, S., Ikeda, Y., Hotomi, M., Yamanaka, N., et al., 2013. Brain regions responsible for tinnitus distress and loudness: a resting-state FMRI study. PLoS One 8, e67778.

van der Loo, E., Gais, S., Congedo, M., Vanneste, S., Plazier, M., Menovsky, T., et al., 2009. Tinnitus intensity dependent gamma oscillations of the contralateral auditory cortex. PLoS One 4, e7396. 1-5.

van der Loo, E., Congedo, M., Vanneste, S., De Heyning, P.V., De Ridder, D., 2011. Insular lateralization in tinnitus distress. Auton. Neurosci. 165, 191-194.

Vanneste, S., De Ridder, D., 2015. Stress-related functional connectivity changes between auditory cortex and cingulate in tinnitus. Brain Connect. 5, 371-383.

Vanneste, S., van de Heyning, P., De Ridder, D., 2011. The neural network of phantom sound changes over time: a comparison between recent-onset and chronic tinnitus patients. Eur. J. Neurosci. 34, 718-731.

Vanneste, S., Song, J.J., De Ridder, D., 2013. Tinnitus and musical hallucinosis: the same but more. Neuroimage 82C, 373-383.

Vanneste, S., Song, J.J., De Ridder, D., 2018a. Thalamocortical dysrhythmia detected by machine learning. Nat. Commun. 9, 1103.

Vanneste, S., Joos, K., Ost, J., De Ridder, D., 2018b. Influencing connectivity and crossfrequency coupling by real-time source localized neurofeedback of the posterior cingulate cortex reduces tinnitus related distress. Neurobiol. Stress 8, 211-224.

Vanneste, S., To, W.T., De Ridder, D., 2019. Tinnitus and neuropathic pain share a common neural substrate in the form of specific brain connectivity and microstate profiles. Prog. Neuropsychopharmacol. Biol. Psychiatry 88, 388-400.

Vielsmeier, V., Lehner, A., Strutz, J., Steffens, T., Kreuzer, P.M., Schecklmann, M., et al., 2015. The relevance of the high frequency audiometry in tinnitus patients with normal hearing in conventional pure-tone audiometry. Biomed. Res. Int. 2015, 302515. 
Vielsmeier, V., Santiago Stiel R., Kwok, P., Langguth, B., Schecklmann, M., 2020. From acute to chronic tinnitus: pilot data on predictors and progression. Front. Neurol. 11, 997.

Wade, J.B., Hart, R.P., 2002. Attention and the stages of pain processing. Pain Med. 3, 30-38.

Wade, J.B., Riddle, D.L., Price, D.D., Dumenci, L., 2011. Role of pain catastrophizing during pain processing in a cohort of patients with chronic and severe arthritic knee pain. Pain $152,314-319$.

Wallhausser-Franke, E., D’Amelio, R., Glauner, A., Delb, W., Servais, J.J., Hormann, K., et al., 2017. Transition from acute to chronic tinnitus: predictors for the development of chronic distressing tinnitus. Front. Neurol. 8, 605.

Weise, C., Hesser, H., Andersson, G., Nyenhuis, N., Zastrutzki, S., Kroner-Herwig, B., et al., 2013. The role of catastrophizing in recent onset tinnitus: its nature and association with tinnitus distress and medical utilization. Int. J. Audiol. 52, 177-188.

Weisz, N., Hartmann, T., Dohrmann, K., Schlee, W., Norena, A., 2006. High-frequency tinnitus without hearing loss does not mean absence of deafferentation. Hear. Res. $222,108-114$.

Wikipedia, 2020a. Theoretical definition. Retrieved 11 February, from https://en.wikipedia. org/wiki/Theoretical_definition\#: :text=From $\% 20$ Wikipedia $\% 2 C \% 20$ the $\% 20$ free $\%$ 20encyclopedia,thinking\%20about\%20potentially\%20related\%20events.

Wikipedia, 2020b. Disorder. Retrieved 08 August, from https://en.wikipedia.org/wiki/ Disease\#Disorder.

Zeman, F., Koller, M., Langguth, B., Landgrebe, M., Tinnitus Research Initiative database study group, 2014. Which tinnitus-related aspects are relevant for quality of life and depression: results from a large international multicentre sample. Health Qual. Life Outcomes 12, 7. 